\title{
Calcifying nanoparticles associated encrusted urinary bladder cystitis
}

\author{
Tomislav M Jelic' \\ Rod Roque' \\ Uzay Yasar ${ }^{2}$ \\ Shayna B Tomchin' \\ Jose M Serrato ${ }^{2}$ \\ Samuel G Deem ${ }^{3}$ \\ James P Tierney ${ }^{3}$ \\ Ho-Huang Chang' \\ 'Department of Pathology \\ Charleston Area Medical Center, \\ Charleston WV, USA; ${ }^{2}$ Urology \\ Center of Charleston, Charleston \\ WV, USA; ${ }^{3}$ Urologic-Surgical Assoc. \\ of Charleston, Charleston WV, USA
}

\begin{abstract}
Encrusted cystitis is a subtype of chronic cystitis characterized by multiple calcifications in the form of plaques located in the interstitium of the urinary bladder mucosa and frequently associated with mucosal ulcers. It is a very rare disease of controversial etiology. Our transmission electron microscopy of the calcified plaques of encrusted cystitis has revealed that the smallest formed particles (elementary units) of these calcifications are electron-dense shells surrounding an electron lucent core, diagnostic of calcifying nanoparticles (previously called nanobacteria). We pioneer the notion that calcifying nanoparticles are the causative agents of encrusted urinary bladder cystitis.
\end{abstract}

Keywords: calcifying nanoparticles, nanobacteria, encrusted cystitis

\section{Introduction}

Encrusted cystitis is a very rare form of chronic cystitis. Calcified plaques composed of calcium salts including phosphate, carbonate, and ammonium-magnesium salts in the bladder mucosa accompanied with inflammation and ulcerations characterize pathologically encrusted cystitis. Despite its increasing incidence in nineties, especially in immunedepressed patients and renal transplant recipients (Meria et al 1998), according to a PubMed search, by spring 2008, only 60 patients have been reported in 60 published articles, mainly single case reports. Etiology of this rare entity, which is characterized by calcifications in the urinary bladder wall, is presently controversial. A few hypotheses exist to explain this phenomenon but are difficult to prove. The most popular of them proposes combined action of mucosal alterations (lesion of any type) and microorganisms that split urea forming ammonia and thus creating an alkaline environment leading to the disease by precipitation of calcium salts (Russell 1901). Corynebacterium group D2 (Fernandez Natal et al 1992), Escherichia coli (Romero Perez et al 1992), Streptoccocus hemolyticus, S. viridians, and Proteus (Jameson 1966) species were denoted as the most frequent culprits. Calcifying nanoparticles (previously called nanobacteria) are self-propagating, cultivable macromolecular complexes (Kajander 2006) generally regarded as bacteria but with a lack of definitive genomic proof. The defining characteristic of calcifying nanoparticles (nanobacteria) is their outer envelope composed of calcium phosphate that presents on transmission electron microscope images as an electron-dense shell surrounding a translucent central core. The name is derived from the very small size of these nanoparticles, which varies from 50 nanometers to 500 nanometers $(\mathrm{nm})$. Calcifying nanoparticles (nanobacteria) are the smallest $(50-500 \mathrm{~nm})$ cultivable replicating agents on earth, which were isolated from bovine and human blood for the first time by Kajander and colleagues (1997) in the last decade of the 20th century. The most important characteristic of nanobacteria is that they produce carbonate apatite on their cell envelope (Kajander and Çiftçioglu 1998) from soluble calcium and phosphorus at physiologic concentrations and conditions. Kajander's discovery initiated further studies and nanobacteria have been implicated in the 
wide array of human diseases associated with calcifications including: kidney stone formation (Çiftçioglu et al 1999), valvular calcifications (Jelic et al 2004; Miller et al 2004), psammoma bodies seen in ovarian cancer (Hudelist et al 2004), calcified atherosclerotic vascular plaques (Miller et al 2004; Puskas et al 2005), cholelithiasis (Wen et al 2005), microcalcification in breast cancer (Altundag et al 2006), calcific aortic valve stenosis (Jelic et al 2007; Bratos-Perez et al 2008), calcifications of placental villi (Agababov et al 2007), and Randall's plaque (Çiftçioglu et al 2008). The etiologic role of nanobacteria in kidney stone formation was confirmed in a small study (Garcia-Cuerpo et al 2000) when nephrolithiais in rats resulted from intrarenal injection of nanobacteria that were previously isolated from kidney stones. Bratos-Perez and colleagues (2008) successfully cultured self-replicating calcifying nanoparticles from the aortic valves of patients with calcific aortic stenosis. Despite all the above, the concept of nanobacteria is still controversial. Some authors interpret nanosize calcifications as precipitation of calcium salts on macromolecules (Cisar et al 1999), or on protein fetuin (Raoult et al 2008) and these and some other authors doubt the mere existence of nanobacteria as living microorganisms (Abott 1999; Urbano and Urbano 2007). This is partially due to the fact that genomic structure of nanobacteria has not yet been elucidated. For this reason Kajander (2006) suggested changing the name of nanobacteria to calcifying nanoparticles, while emphasizing that they are self-propagating, cultivable, and infectious agents. Recently Mathew and colleagues (2008) documented that calcifying nanoparticles were self-replicators in physiological conditions and not simple crystals of precipitated inorganic apatite. We have found evidence for an association between calcifying nanoparticles and encrusted cystitis.

\section{Case report}

The patient is a 43-year-old man, a heavy smoker (3-4 packs a day for over 10 years) who presented with complaints of hematuria and low back pain. He was found to have multiple recurring papillary noninvasive urothelial (transitional) cell carcinomas at multiple bladder sites grade 1 and grade 2 (in a scale $1-4$ ) that were completely removed during a 6-month period. He had three separate transurethral resections of the tumors performed approximately every three months. After the second resection (July 2006) a single instillation of 40 milligrams of mitomycin $\mathrm{C}$ was administered. Four months later he received six courses of Bacillus CalmetteGuerin (BCG) over a six-week therapy to prevent bladder tumor recurrence per current recommended guidelines.
Calcium and creatinine blood concentrations were normal $9.2 \mathrm{mg} / \mathrm{dl}$ and $0.7 \mathrm{mg} / \mathrm{dl}$, respectively. The patient's urine did not smell of ammonia. The urine was acidic with a $\mathrm{pH}$ of 5. The specific gravity was 1.019. Nitrites were negative and sediment showed 3 erythrocytes and 23 leukocytes. Standard (not prolonged) urine culture was negative. The patient was not treated with antibiotics or uroantiseptics except during cystoscopy procedure to prevent iatrogenic infection. Cystoscopy performed 3 months after the second surgery and before BCG application identified the recurrent papillary tumor. It also visualized multiple calcified plaques (Figure 1) in the bladder dome. These plaques were associated with at least two ulcerations that were hard on palpation due to these calcifications. The larger ulcer was actively bleeding prior to any manipulation. The small papillary tumor was extirpated and the calcifications were removed from the dome piece by piece. Repeated bladder biopsy 6 months later showed no evidence of malignancy but encrusted cystitis was still present.

\section{Material and methods}

Biopsies of the tumor and of the bladder dome were fixed in $10 \%$ buffered formaldehyde neutral solution and embedded into paraffin blocks. Paraffin blocks were cut on standard rotary microtomes with a routine setting of 4 microns. One ribbon from each paraffin block was placed on glass slide in order to be stained with hematoxylin and eosin. The staining procedure was performed by an automated robotic stainer with a line up of solutions as follows: Biocleer-F, absolute alcohol, 95\% and 80\% alcohol, Gill's III Hematoxylin (7 minutes), running tap water, $1 \%$ acid alcohol, and eosin ( $1 \mathrm{~min}$ ), row of alcohol solutions, and finally Biocleer-F. After staining the glass slide was placed in a xylene rinse to activate the cover

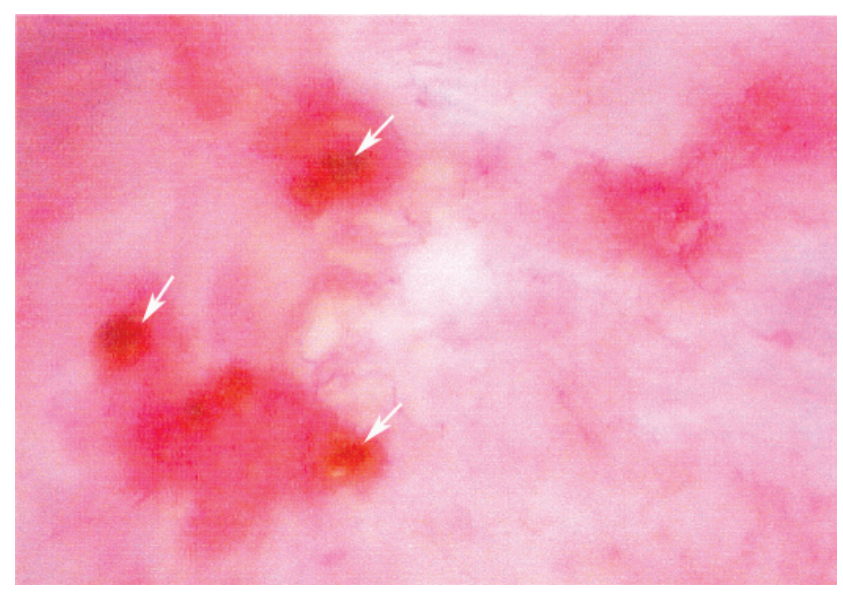

Figure I Calcifying plaques (white arrows) of encrusted cystitis, cystoscopy photograph. 
slip adhesion on the automated cover-slipping machine. Von Kossa's method for calcium staining is based on silver salt and was performed as follows: ribbon 6 microns thin was cut off paraffin block by standard rotary microtome and placed on glass slide. After deparaffinization and hydration with distilled water the glass slide was placed in 5\% silver nitrate solution for 60 minutes exposed to ultraviolet lamp. After rinsing in distilled water glass slide was placed in 5\% sodium thiosulfate (hypo) solution for 2 minutes. After rinsing in distilled water nuclear and cytoplsmic counterstaining was performed in fast red solution (eosin) for 5 minutes. Rinsing in distilled water, dehydration in alcohol, clearing in xylene and mounting with Permount were final steps in von Kossa's procedure for calcium staining. Glass slides were examined through a light microscope and color photographs were taken. Standard procedure for obtaining electron microcope images was implemented as follows: the calcified area was cut out from the paraffin block, deparaffinized before embedding in a polymerized block of spur epoxy resin, and cut into 600 to 900 angstroms thin slices that were stained with uranyl acetate stain and lead citrate stain. Transmission electron microscopy was performed on a Zeiss model CEM 902 instrument manufactured in West Germany.

\section{Results}

Light microscopic examination of the hematoxylin and eosin stained sections of the tumor showed noninvasive, low-grade papillary urothelial carcinoma. Biopsies from the bladder ulcers showed encrusted cystitis with an abundance of calcifications confirmed by von Kossa stain (Figure 2 A-C).

Transmission electron microscopy of the encrusted cystitis calcifications revealed that the smallest constituents of calcifications were not amorphous substance or granules but formed particles (elementary units of calcifications), composed of electron-dense shell rimming a central translucent core. They vary in size (Figures 3,4) from $165 \mathrm{~nm}$ to $440 \mathrm{~nm}$ and exhibit the size and spherical-to-ovoid shape diagnostic of calcifying nanoparticles/nanobacteria according to the relevant literature and our experience. It is easy to notice in Figure 3 that merging of the calcifying nanoparticles (like stacking of snowballs) forms micron-size calcium aggregates (micron-size plaques) measuring about 3 square microns

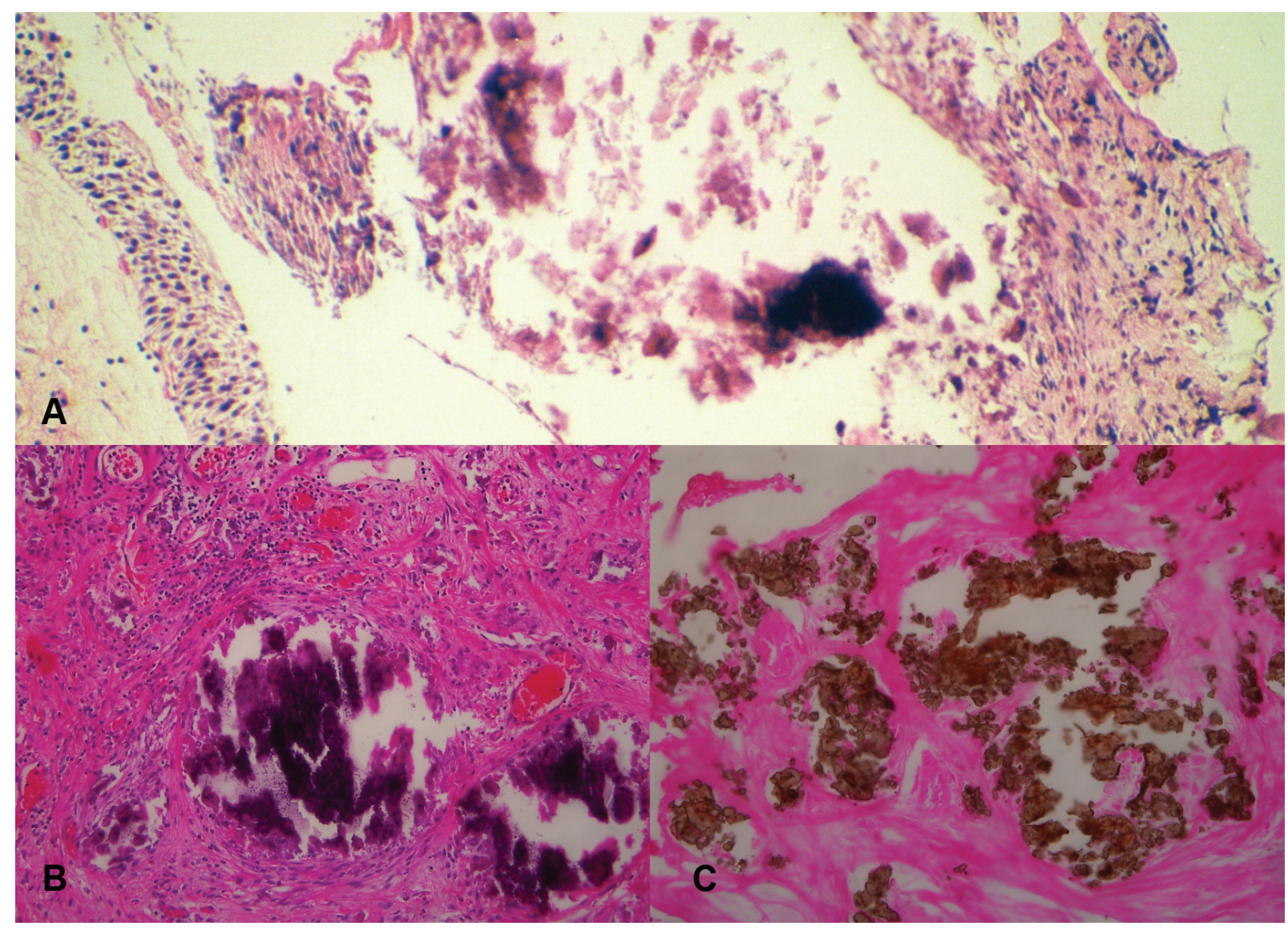

Figure 2 Light microscopy composed picture showing encrusted cystitis, magnification $100 \times$, hematoxylin and eosin, A-calcifications in the interstitium of the urothelial mucosa, B-calcifications and chronic inflammatory cells in the interstitium, C-calcium stained brown by von Kossa stain. 


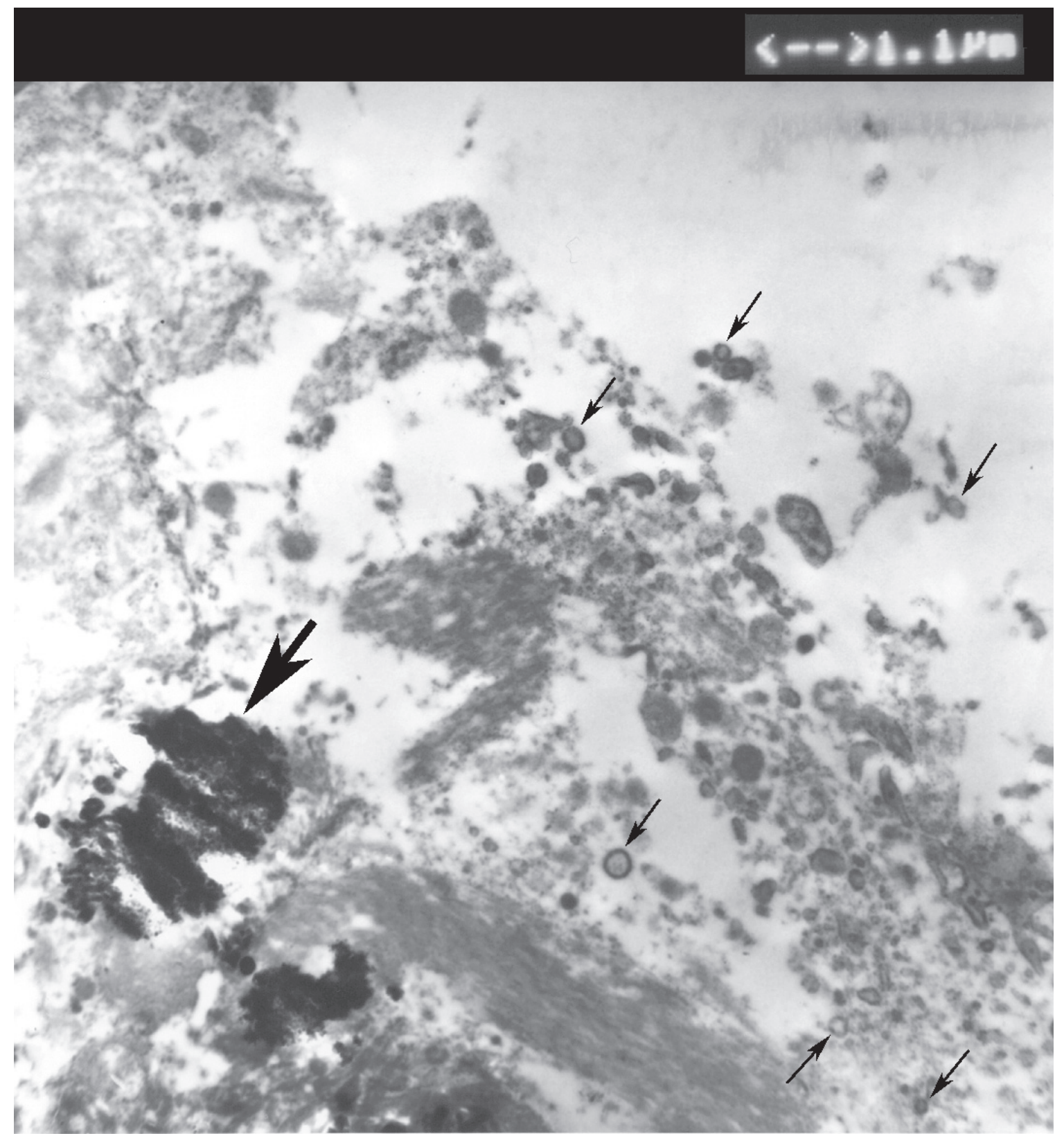

Figure 3 Transmission electron microscopy image of encrusted cystitis calcifications (magnification 7,000) demonstrating multiple calcifying nanoparticles (some indicated by black thin arrows) with the characteristic electron-dense shell (ring structures $165 \mathrm{~nm}$ to $440 \mathrm{~nm}$ ) surrounding a central electron lucent core. A thick black arrow points to the micron-size calcium aggregate about 3 square microns.

that by further merging have ultimately become plaques of encrusted cystitis visible by light microscope (Figure 2) and naked eye (Figure 1).

\section{Discussion}

Our electron microscopic study of the calcifications in the urinary bladder mucosa of the patient with encrusted cystitis revealed that the smallest formed structures, to wit elementary units of these calcifications are electron-dense rings with central lucent core diagnostic of calcifying nanoparticles. As discussed in length in the introduction, controversy about nature of calcifying nanoparticles (previously called nanobacteria) will continue in the future.

Encrusted cystitis is a very rare form of cystitis still of controversial etiology and thus no standardized therapy exists, although the first of report for cure of an individual patient appeared 107 years ago (Russell 1901). Papillary urothelial carcinomas in our patient were successfully eradicated but removal of calcified plaques was only partial and encrusted cystitis persisted. Prevailing view is that 


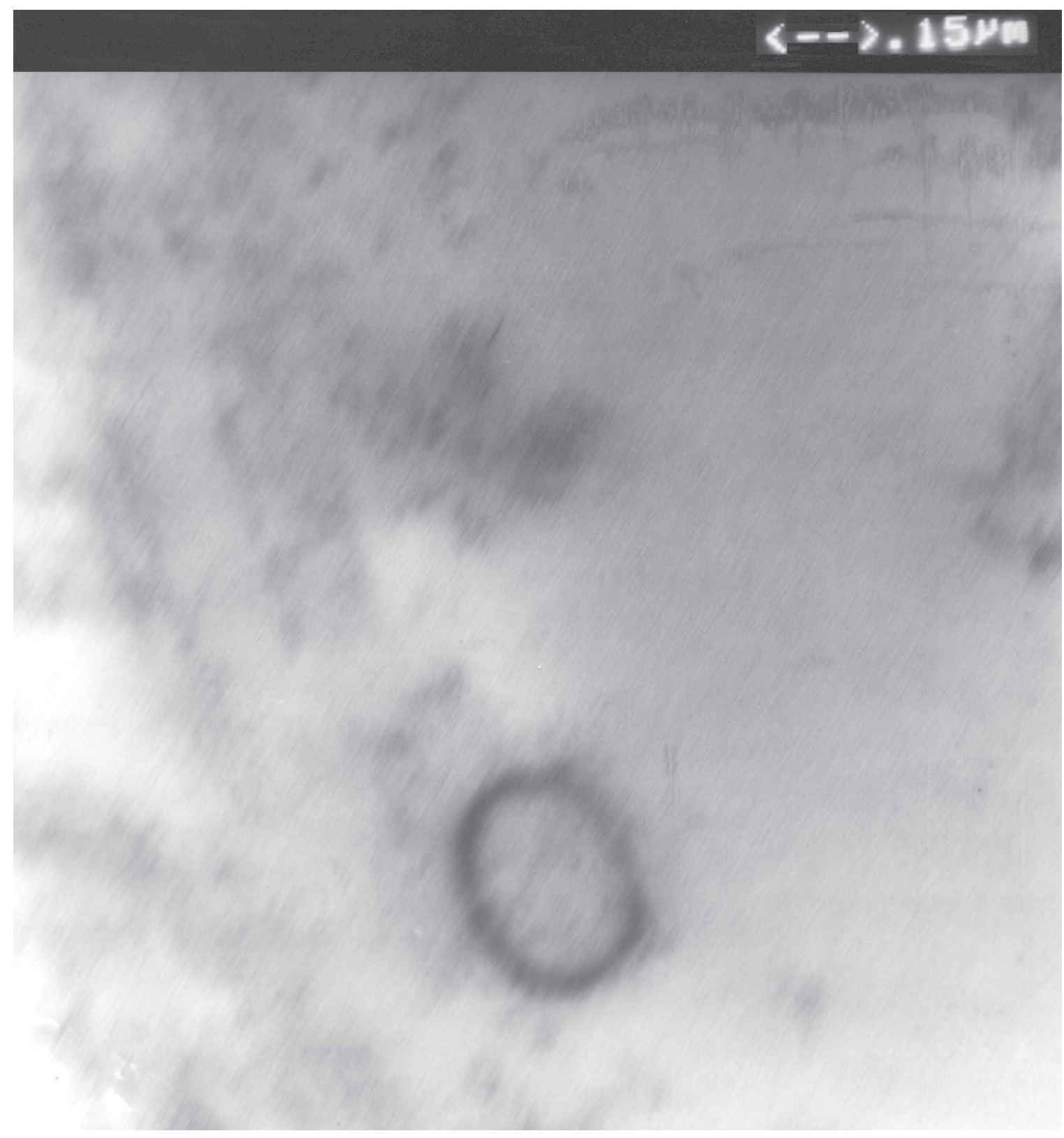

Figure 4 Calcyifying nanoparticle $(280 \mathrm{~nm} \times 230 \mathrm{~nm})$.Transmission electron microscopy image (magnification 50,000) presenting characteristic electron-dense shell surrounding an electron lucent core.

encrusted cystitis is alkaline cystitis due to urea splitting bacteria (mainly Corynebacterium group D2). However, it was found later (Huget-Perez et al 1999) that not all patients with encrusted cystitis have alkaline urine implying that other mechanisms for calcifications may play role in the genesis of encrusted cystitis. Our patient had acidic urine with a $\mathrm{pH}$ of 5 and standard urine culture (not prolonged) for common microorganisms showed no growth. It has not been documented in the literature that nanobacteria or calcifying nanoparticles can split urea. It has been demonstrated in vitro (Çiftçioglu et al 2008) that nanobacteria (calcifying nanoparticles) were inhibited by tetracycline, nitrofurantoin, trimethoprim, trimethoprim-sulfamethoxazole, and ampicillin at levels achievable in serum and urine. Our patient was not treated with any of these drugs. Encrusted cystitis has also been reported after the intravesical application of BCG (Spirnak et al 1993) or mitomycin C (Alter and Malek 1987) for the treatment of bladder carcinoma. Our patient developed encrusted cystitis before BCG application. Since the first report by Alter and Malek (1987), only 6 additional cases of intravesical calcifications after local application of mitomycin $\mathrm{C}$ were reported, it is unlikely that intravesical 
instillation of this cytostatic drug in our patient caused encrusted cystitis but it cannot be totally excluded. At this point in time, when nature of calcifying nanoparticles is unresolved and their bacterial structure uncertain, it is not warranted to speculate that mitomycin $\mathrm{C}$ facilitated infection with calcifying nanoparticles/nanobacteria. We have revealed by electron microscopic study calcifying nanoparticles in the calcifications of the encrusted cystitis. From this finding we have inferred that calcifying nanoparticles are associated with encrusted cystitis and are quite possibly the causative agents of this very rare disease. At this point in time with scant knowledge of pathogenesis and epidemiology of calcifying nanoparticles, a discussion about the mode of infection or their access to bladder mucosa is premature. Further studies are necessary in order to confirm whether calcifying nanoparticles are causative agents of encrusted cystitis or only its pathologic marker.

\section{Disclosure}

The authors report no conflicts of interest in this work.

\section{Acknowledgment}

We appreciate the courtesy of James R. Angel, M.D. from Campbellsville, Kentucky, USA.

\section{References}

Abott A. 1999. Battle lines drawn between "nanobacteria" researchers. Nature, 401:105.

Agababov RM, Abashina TN, Suzina NE, et al. 2007. Link between the early calcium deposition in placenta and nanobacterial-like infection. J Biosci, 32:1163-68.

Alter AJ, Malek GH. 1987. Bladder wall calcification after topical mitomycin C. J Urol, 138:1239-40.

Altundag K, Altundag O, Akyurek S, et al. 2006. Possible association between nanobacteria and malignant microcalcifications in breast cancer. Breast J, 12:287.

Bratos-Perez MA, Sanchez PL, De Cruz SG, et al. 2008. Association between self-replicating calcifying nanoparticles and aortic stenosis: a possible link to valve calcification. Eur Heart J, 29:371-6.

Çiftçioglu N, Bjorklund M, Kuorikoski K, et al. 1999. Nanobacteria: an infectious cause for kidney stone formation. Kidney Int, 56:1893-8.

Çiftçioglu N, Vejdani K, Lee O, et al. 2008. Association between Randall's plaque and calcifying nanoparticles. Int J Nanomedicine, 3:105-15.

Çiftçioglu N, Miller-Hjelle MA, Hjelle JT, et al. 2002. Inhibition of nanobacteria by antimicrobial drugs as measured by modified microdilution method. Antimicrob Agents Chemother, 46:2077-86.
Cisar JO, Xu DQ, Thompson J, et al. 2000. An alternative interpretation of nanobacteria-induced biomineralization. Proc Natl Acad Sci USA, 21:11511-15.

Fernandez Natal MI, Garcia Diez F, Salas Valien JS, et al. 1992. Incrusetd cystitis with isolation of Corynebacterium group D2. Med Clin (Barc), 98:419-22.

Garcia-Cuerpo E, Kajander EO, Çiftçioglu N, et al. 2000. [Nanobacteria; Un modelo de neo-litogenesis experimental] Arch Esp Urol, 53:291-303.

Hudelist G, Singer CF, Kubista E, et al. 2004. Presence of nanobacteria in psammoma bodies of ovarian cancer: evidence for pathogenetic role in intratumoral biomineralization. Histopathology, 45:633-7.

Huget-Perez J, Salvador-Bayarri J, Vicente-Rodriguez J. 1999. Encrusted cystitis. Is it always alkaline? Arch Esp Urol, 52:157-64.

Jameson RM. 1966. The treatment of phosphatie encrusted cystitis (alkaline cystitis) with nalidixic acid. Br J Urol, 38:89-92.

Jelic TM, Malas AM, Groves SS, et al. 2004. Nanobacteria-caused mitral valve calciphylaxis in a man with diabetic renal failure. South Med J, 97:194-8.

Jelic TM, Chang HH, Roque R, et al. 2007. Nanobacteria-associated calcific aortic valve stenosis. J Heart Valve Dis, 16:101-5.

Kajander EO, Kuronen I, Akerman KK, et al. 1997. Nanobacteria from blood, the smallest culturable autonomously replicating agent on earth. Proc SPIE, 3111:420-8.

Kajander EO, Çiftçioglu N. 1998. Nanobacteria: an alternative mechanism for pathogenic intra- and extracellular calcification and stone formation. Proc Natl Acad Sci USA, 95:8274-9.

Kajander EO. 2006. Nanobacteria-propagating calcifying nanoparticles. Lett Appl Microbiol, 42:367-79.

Mathew G, McKay DS, Çiftçioglu N. 2008. Do blood-borne calcifying nanoparticles self-propagate? Int J Nanomedicine, 3(2): In press.

Meria P, Desgrippes A, Arfi C, et al. 1998. Encrusted cystitis and pyelitis. J Urol, 160:3-9.

Miller VM, Rodgers G, Charlesworth JA, et al. 2004. Evidence of nanobacterial - like structures in calcified human arteries and cardiac valves. Am J Physiol Heart Circ Physiol, 287:H1115-24.

Puskas LG, Tiszlavicz L, Razga Z, et al. 2005. Detection of nanobacterialike particles in human atherosclerotic plaques. Acta Biol Hung, 56:233-45.

Raoult D, Drancourt M, Azza S, et al. 2008. Nanobacteria are mineralo fetuin complexes. PloS Pathog, 4:e41.

Romero Perez P, Amat Cecilia M, Omera Arbash AR, et al. 1992. Encrusted cystitis. Review of the literature and report of case. Actas Urol Esp, 16:496-505.

Russell AW. 1901. Notes of the progress of cure of a case of persistent cystitis with phosphatic concretions. Glasgow Med J, 55:376-8.

Spirnak JP, Lubke WL, Thompson IM, et al. 1993. Dystrophic bladder wall calcifications following intravesical BCG treatment for superficial transitional cell carcinoma of bladder. Urology, 42:89-92.

Urbano P, Urbano F. 2007. Nanobacteria: facts or fancies? PLoS Pathog, 3:e55.

Wen Y, Li YG, Yang ZL, et al. 2005. Detection of nanobacteria in serum, bile and gallbladder mucosa of patients with cheolecystolithiasis. Chin Med J, 118:421-4. 